\title{
Derived neutrophil lymphocyte ratio is predictive of survival from intermittent therapy in advanced colorectal cancer: a post hoc analysis of the MRC COIN study
}

\begin{abstract}
Tal Grenader ${ }^{1}$, Stephen Nash ${ }^{2}$, Richard Adams ${ }^{3}$, Richard Kaplan ${ }^{4}$, David Fisher ${ }^{4}$, Tim Maughan $^{5}$ and John Bridgewater ${ }^{\star}, 6$

${ }^{1}$ Oncology Institute, Shaare Zedek Medical Centre, Serusatem, Israel; ${ }^{2}$ Cancer Research UK \& UCL Cancer Trials Centre, London WIT 4TJ4TJ, UK; ${ }^{3}$ Institute of Cancer \& Genetics, Cardiff University School of Medicine Velindre Hospital, Cardiff CF14 2TL, UK; ${ }^{4}$ MRC Clinical Trials Unit at UCL, London WC2B 6NH, UK; ${ }^{5}$ CRUK/MRC Oxford Institute for Radiation, Oxford OX3 7DQ, UK and ${ }^{6}$ UCL Cancer Institute, London WC1E 6DD, UK
\end{abstract}

Background: The phase III COntinuous or INtermittent (COIN) trial failed to show non-inferiority of intermittent compared with continuous chemotherapy for advanced colorectal cancer in overall survival (OS). The present analysis evaluated whether the derived neutrophil to lymphocyte ratio (dNLR) could predict the effect of intermittent vs continuous chemotherapy on OS in patients with advanced colorectal cancer.

Methods: A post hoc exploratory analysis of COIN arms $A$ and $C$ was performed. Landmark analysis was conducted on all patients with available WBC and neutrophils data. The dNLR was calculated using a formula which has previously demonstrated predictive power in cancer patients: $d N L R=A N C /(W B C-A N C)$. A high $d N L R$ was defined using a cut-off value of $\geqslant 2.22$. Derived neutrophil to lymphocyte ratio was then correlated with clinical outcomes. Survival curves were generated based on dNLR using the KaplanMeier method. Comparison between groups was performed using Cox regression.

Results: A total of 1630 patients were assigned to the continuous $(N=815)$ or intermittent $(N=815)$ arms. There was a strong association between dNLR level and OS. The median survival times in the ITT population were 18.6 months and 12.5 months for patients with low and high $\mathrm{dNLR}$, respectively $(\mathrm{HR}=1.70 ; 95 \% \mathrm{Cl}=1.52-1.90 ; P<0.001)$. The estimate of the hazard ratio did not alter substantially $(H R=1.54)$ after adjusting for treatment, tumour status, number of metastatic sites, alkaline phosphate and platelet count.

Conclusions: Derived neutrophil to lymphocyte ratio is strongly prognostic for survival in the COIN intermittent vs continuous treatment arms. Derived neutrophil to lymphocyte ratio does not predict for detrimental survival in patients treated with intermittent therapy.

The treatment of advanced colorectal cancer has improved substantially during the past decade with the introduction of new and more effective drugs and advances in our understanding of the disease's molecular biology (Douillard et al., 2013). However, the balance between improved survival and costs, in terms of toxicity, quality of life and financial, can be difficult. The COIN trial (COntinuous or INtermittent) was developed to conclusively address this issue (Adams et al., 2011). Approximately 1600 patients with advanced colorectal cancer were randomised to receive intermittent $v s$ continuous systemic therapy with

*Correspondence: Professor J Bridgewater; E-mail: j.bridgewater@ucl.ac.uk

Received 9 September 2015; revised 24 November 2015; accepted 4 January 2016; published online 18 February 2016

(c) 2016 Cancer Research UK. All rights reserved 0007-0920/16 
oxaliplatin and fluoropyrimidine. Although the trial did not show non-inferiority of intermittent compared with continuous chemotherapy for advanced colorectal cancer in terms of overall survival, a subgroup analysis suggests that patients with normal baseline platelet counts could gain the benefits of intermittent chemotherapy without detriment in survival, whereas those with raised baseline platelet counts have impaired survival and quality of life with intermittent chemotherapy, and may do better without a treatment break.

Numerous studies have established that elevated inflammatory markers, such as C-reactive protein, neutrophil/lymphocyte ratio (NLR) and platelet-lymphocyte ratio have been associated with poor outcomes in cancer patients with colorectal cancer (Walsh et al., 2005; Liu et al., 2010; Proctor et al., 2012). Platelets count and neutrophil lymphocyte ratio are inexpensive markers of host inflammation, and may reflect cytokine activation and more aggressive disease. A recently reported meta-analysis of 100 studies comprising 40559 patients found NLR > 4 was associated with poorer OS with hazard ratio for OS of 1.81 (95\% CI =1.67-1.97; $P<0.001)$, an effect observed in all disease subgroups, sites, and stages (Templeton et al., 2014). Lymphocyte count data was not collected in the COIN trial, however the derived NLR (dNLR) has been shown to possess similar prognostic value (Dirican et al., 2014).

The aim of the present correlative analysis was to evaluate whether dNLR could predict the effect of intermittent $v s$ continuous first-line oxaliplatin and fluoropyrimidine chemotherapy on overall survival in patients with advanced colorectal cancer.

\section{MATERIALS AND METHODS}

The trial design and eligibility criteria have been reported previously (Adams et al., 2011). The primary objective of the phase 3 MRC COIN study was to assess the effect of pre-planned treatment interruptions in oxaliplatin and fluoropyrimidine combination chemotherapy on overall survival. A second randomisation in the trial assessed the effect of addition of cetuximab to continuous oxaliplatin and fluoropyrimidine combination chemo therapy.

After enrolment, patients were randomly assigned (1) to the control arm of continuous (arm A) or intermittent (arm C) chemotherapy. In arm A treatment was continued until disease progression, development of cumulative toxic effects, or patient choice. Patients on arm $\mathrm{C}$ received chemotherapy for 12 weeks, after which treatment was stopped completely and the patients were assessed with imaging every 12 weeks. The same chemotherapy was restarted on progression.

Derived neutrophil/lymphocyte ratio (dNLR) calculation. White blood cell (WBC) and absolute neutrophil count (ANC) were obtained at patient enrolment. The dNLR was calculated using a formula that was previously shown to have predictive power in cancer patients (Walsh et al., 2005; Dirican et al., 2014).

Statistical methods. All statistical analyses were performed by the Cancer Research UK and University College London Cancer Trials Centre. All randomly assigned patients for whom data on WBC and ANC in patient enrolment were available were included in the analysis. A high dNLR was defined using a cut-off value of 2.2, which was the median dNLR.

Associations were assessed using Kaplan-Meier plots and Cox proportional hazard models. An interaction between dNLR $(<2.22)$ and treatment allocation was used to assess the difference in treatment effects between the two trial arms. Models were adjusted for the a priori factors of treatment arm (standard, intermittent), tumour status (resected, locally recurrent, unresected), number of metastatic sites ( 0 or 1,2 or more), CEA at baseline $\left(<100, \geqslant 100 \mathrm{mgl}^{-1}\right)$, alkaline phosphatase at baseline $\left(<100, \geqslant 100 \mathrm{Ul}^{-1}\right)$ and platelet count at baseline $\left(<400000 \mu \mathrm{l}^{-1}, \geqslant 400000 \mu \mathrm{l}^{-1}\right)$. Factors were excluded from the model when they were missing for more than $10 \%$ of the population including CEA. Initial analysis of the association between dNLR and overall survival was performed on the ITT population; all comparisons between treatment groups was performed on both the ITT and the per protocol population, defined as all patients who reached the point where the continuous and intermittent strategies diverged, consistent with the original trial definition. As this was a non-inferiority trial, all comparisons between the treatment groups are reported with $80 \%$ confidence intervals, with a boundary of 1.162 used to assess noninferiority (Adams et al., 2011). Receiver operating characteristic analysis was performed to calculate area under the curve (AUC) using one-year survival as the outcome and baseline dNLR as the test variable.

Statistical analysis was carried out using Stata version 12.1 (College Station, TX, USA).

\section{RESULTS}

Population studied. A total of 1630 patients were assigned to the continuous $(N=815)$ or intermittent $(N=815)$ arms. There was incomplete data for 32 patients $(N=8$ for missing dates, $N=55$ for missing platelets, $N=1313$ for missing dNLR) who were excluded, resulting in a cohort of 1604 patients (accounting for $98.4 \%$ of the total study population). Of these patients, 971 (60.5\%) remained on study beyond 12 weeks and were included in the per protocol analysis (Table 1).

Prognostic. There was a strong association between dNLR level and overall survival. The median survival times in the ITT population were 18.6 months and 12.5 months for patients with low and high dNLR, respectively $(\mathrm{HR}=1.70 ; 95 \% \mathrm{CI}=1.52-1.90$; $P<0.001)$. The estimate of the hazard ratio did not alter substantially $(\mathrm{HR}=1.54)$ after adjusting for treatment, tumour status, number of metastatic sites, alkaline phosphatase and platelet count (Figure 1). The AUC was $64.5 \%$ and there was a detection rate for survival of $82.5 \%$ and a false positive rate of $63.1 \%$. As a comparator, the AUC for platelets was $62.8 \%$, and using a value of $400000 \mu \mathrm{l}^{-1}$ to dichotomise baseline platelets there was a detection rate of $76.8 \%$ and a false positive rate of $58.8 \%$. Both criteria remain modestly prognostic as previously defined (Walter, 2002).

Predictive effect of high dNLR. There was no evidence of a differential effect of treatment between the two dNLR groups in the ITT population only $(P=0.20)$. Among patients with low baseline dNLR, the hazard ratio for treatment allocation was $1.16(80 \%$ $\mathrm{CI}=1.00-1.34)$ compared to $1.21(80 \% \mathrm{CI}=1.09-1.34)$ among the high dNLR group. In the per protocol population $(N=971)$, the hazard ratios were 1.06 and 1.26 for the low and high dNLR groups respectively. There was no evidence to support a differential treatment effect in this population $(P=0.26$ for interaction between dNLR group and treatment allocation), nor evidence of non-inferiority (Figure 2 and Table 2).

As previously reported, a raised platelet count at baseline $\left(\geqslant 400000 \mu \mathrm{l}^{-1}\right.$, recorded for $271(28 \%)$ of 978 patients) predicts a significant survival detriment from intermittent chemotherapy ( $P=0.0027$ for interaction). Combining platelet count and dNLR as a single predictive factor was not superior to platelet count alone (Supplementary Table 1). 
Table 1. Population demographics by dNLR

\begin{tabular}{|c|c|c|}
\hline & $\begin{array}{l}\mathrm{dNLR} \leqslant 2.2 \\
(N=805)\end{array}$ & $\begin{array}{c}\mathrm{dNLR} \geqslant 2.2 \\
(N=799)\end{array}$ \\
\hline \multicolumn{3}{|l|}{ Treatment } \\
\hline A-Standard chemotherapy & $391(48.6)$ & $408(51.1)$ \\
\hline C-Intermittent chemotherapy & $414(51.4)$ & 391 (48.9) \\
\hline Age; median (range) & $63.8(18-82)$ & $63.6(25-87)$ \\
\hline \multicolumn{3}{|l|}{ Sex } \\
\hline Male & $538(66.8)$ & $499(62.5)$ \\
\hline Female & $267(33.2)$ & $300(37.5)$ \\
\hline \multicolumn{3}{|l|}{ WHO performance status } \\
\hline 0-Normal activity without restriction & $417(51.8)$ & $320(40.1)$ \\
\hline & $348(43.2)$ & $402(50.3)$ \\
\hline $\begin{array}{l}2-U p \text { and about }>50 \% \text { of waking } \\
\text { hours, limited self-care }\end{array}$ & $40(5.0)$ & $77(9.6)$ \\
\hline \multicolumn{3}{|l|}{ Status of primary tumour } \\
\hline Resected & $485(60.2)$ & $364(45.6)$ \\
\hline Local recurrence & $40(5.0)$ & $44(5.5)$ \\
\hline Unresected/unresectable & $280(34.8)$ & $391(48.9)$ \\
\hline \multicolumn{3}{|l|}{ Number of metastatic sites } \\
\hline 0 & $5(0.6)$ & $8(1.0)$ \\
\hline 1 & $295(36.6)$ & $262(32.8)$ \\
\hline 2 & $326(40.5)$ & $316(39.5)$ \\
\hline $3+$ & $179(22.2)$ & $213(26.7)$ \\
\hline \multicolumn{3}{|l|}{ Liver-only metastases } \\
\hline No & $623(77.4)$ & $634(79.3)$ \\
\hline Yes & $182(22.6)$ & $165(20.7)$ \\
\hline \multicolumn{3}{|l|}{ Platelets } \\
\hline$<400000 \mu 1^{-1}$ & $633(78.6)$ & $490(61.3)$ \\
\hline$\geqslant 400000 \mu \mathrm{l}^{-1}$ & $172(21.4)$ & $309(38.7)$ \\
\hline \multicolumn{3}{|l|}{ CEA } \\
\hline$<100$ & $402(65.0)$ & $319(50.2)$ \\
\hline$\geqslant 100$ & $216(35.0)$ & $316(49.8)$ \\
\hline Missing & 187 & 164 \\
\hline \multicolumn{3}{|l|}{ Alkaline phosphatase } \\
\hline$<300$ & $715(88.8)$ & $620(77.6)$ \\
\hline$\geqslant 300$ & $90(11.2)$ & $179(22.4)$ \\
\hline \multicolumn{3}{|c|}{ Mutations of KRAS, NRAF \& BRAF } \\
\hline All wild-type & $299(46.9)$ & $293(47.7)$ \\
\hline At least one mutation & $338(53.1)$ & $321(52.3)$ \\
\hline Missing & 168 & 185 \\
\hline Included in per-protocol analysis & $519(64.5)$ & $452(56.6)$ \\
\hline
\end{tabular}

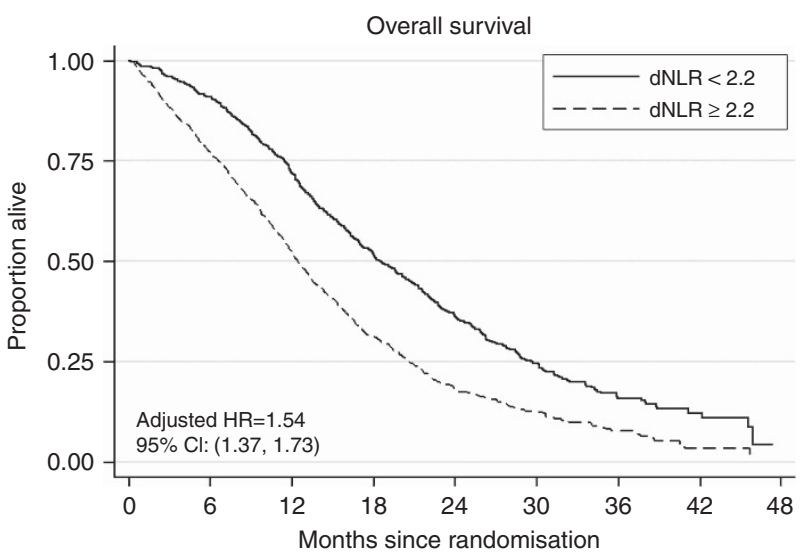

Number at risk

$\begin{array}{llllllllll}\mathrm{dNLR}<2.2 & 805 & 729 & 568 & 358 & 194 & 97 & 39 & 9 & 0\end{array}$ $\begin{array}{llllllllll}\mathrm{dNLR} \geq 2.2 & 799 & 614 & 409 & 212 & 100 & 49 & 19 & 4 & 1\end{array}$

Figure 1. Prognostic impact of dNLR on overall survival.
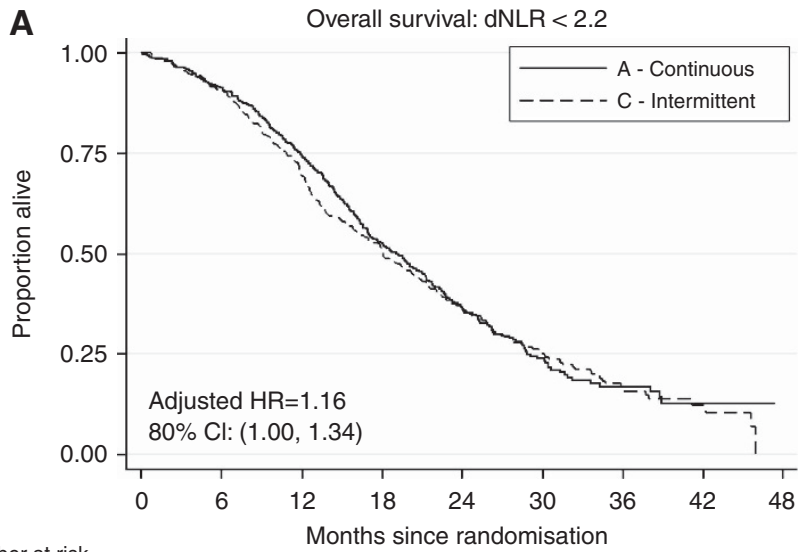

$\begin{array}{llllllllll}\text { A - Continuous } & 391 & 355 & 285 & 178 & 92 & 43 & 17 & 2 & 0 \\ \text { C - Intermittent } & 414 & 374 & 283 & 180 & 102 & 54 & 22 & 7 & 0\end{array}$

\section{B Overall survival: $\mathrm{dNLR} \geq 2.2$}

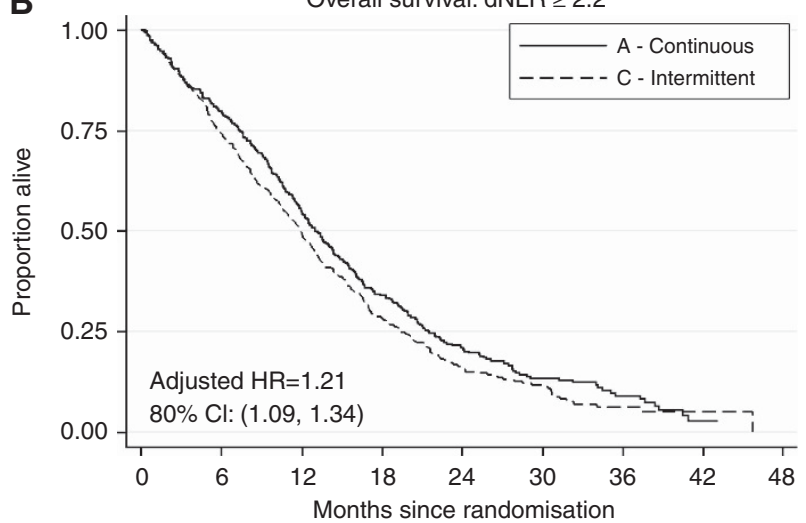

Number at risk

$\begin{array}{llllllllll}\text { A - Continuous } & 408 & 326 & 220 & 116 & 55 & 28 & 13 & 2 & 1\end{array}$

$\begin{array}{llllllllll}\text { C-Intermittent } & 391 & 288 & 189 & 96 & 45 & 21 & 6 & 2 & 0\end{array}$

Figure 2. (A) Impact of low dNLR on overall survival. (B) Impact of high dNLR on overall survival.

Table 2. Hazard ratios and median survival times of ITT and per protocol populations by $\mathrm{dNLR}^{\mathrm{a}}$

\begin{tabular}{|c|c|c|c|}
\hline Population & $\begin{array}{c}\text { Median survival time } \\
\text { (Arm A vs Arm C) }\end{array}$ & HR $(80 \% \mathrm{Cl})$ & $\begin{array}{l}P \text {-value for } \\
\text { interaction }\end{array}$ \\
\hline \multicolumn{4}{|l|}{ ITT } \\
\hline $\begin{array}{l}d N L R<2.2 \\
(N=805) \\
d N L R \geqslant 2.2 \\
(N=799)\end{array}$ & $\begin{array}{l}19.2 \text { vs } 18.1 \\
13.0 \text { vs } 11.9\end{array}$ & $\begin{array}{l}1.04(0.936-1.163) \\
1.21(1.094-1.335)\end{array}$ & 0.20 \\
\hline \multicolumn{4}{|l|}{ Per protocol } \\
\hline $\begin{array}{l}d N L R<2.2 \\
(N=519) \\
d N L R \geqslant 2.2 \\
(N=452)\end{array}$ & $\begin{array}{l}21.8 \text { vs } 21.5 \\
16.6 \text { vs } 15.4\end{array}$ & $\begin{array}{l}1.06(0.921-1.224) \\
1.26(1.099-1.446)\end{array}$ & 0.26 \\
\hline
\end{tabular}

\section{DISCUSSION}

We demonstrate that dNLR is prognostic in the COIN intermittent $v s$ continuous comparison but does not add to the platelet count in selecting patients who would benefit from continuous rather than intermittent therapy. Although some cut-off value of $\geqslant 2.2$ did select a population who benefited from continuous treatment, such an approach lacks robust statistical process and we have reported 
an outcome using the median dNLR of 2.2. We also conclude that the interpretation of $\mathrm{dNLR}$ and the platelet count as predictive factors is more complex than these numerical values would suggest.

A series of studies have proposed that inflammation-based prognostic systems like neutrophil to lymphocyte ratio and thrombocytosis are associated with poor survival of subjects with cancer. A systematic review of 100 studies comprising 40559 patient with various solid tumours found NLR was associated with an adverse OS hazard ratio of $1.81(95 \% \mathrm{CI}=1.67-1.97 ; P<0.001)$ (Templeton et al., 2014), an effect observed in all disease subgroups, sites, and stages. In the six prospective studies used in the NLR meta-analysis containing patients with metastatic colorectal cancer, there were a total of 1817 patients.

The biological mechanism responsible for a higher dNLR requires further investigation, particularly in the context of clinical studies of ruxolitinib and the checkpoint inhibitors. A platelet count of $>400$ remains the most robust criteria by which to select patients for intermittent therapy, and this is being investigated in the FOCUS-4 study (http://www.focus4trial.org/). Statistical uncertainties with respect to biomarker cut-off values continue to generate difficulties in the interpretation of prognostic and predictive biomarkers.

In conclusion, both dNLR and platelets counts were prognostic in patients with metastatic colorectal cancer receiving first-line chemotherapy in randomised phase III COIN trial. A high dNLR $\geqslant 2.22$ and platelets $\geqslant 400000 \mu \mathrm{l}^{-1}$ were associated with shorter survival. However, in contrast to platelet count, a high dNLR failed to predict inferiority of intermittent chemotherapy and cannot be used for clinical decision of which patients can benefit from chemotherapy-free breaks.

\section{ACKNOWLEDGEMENTS}

TG is a fellow of the European Society of Medical Oncology. SN was supported by CRUK Grant C444/A15953 to the UCL CRUK Trials Centre. JB is partly supported by the UCLH/UCL Biomedical Research Centre.

\section{CONFLICT OF INTEREST}

The authors declare no conflict of interest.

\section{REFERENCES}

Adams RA, Meade AM, Seymour MT, Wilson RH, Madi A, Fisher D, Kenny SL, Kay E, Hodgkinson E, Pope M, Rogers P, Wasan H, Falk S, Gollins S, Hickish T, Bessell EM, Propper D, Kennedy MJ, Kaplan R, Maughan TS. MRC COIN Trial Investigators (2011) Intermittent versus continuous oxaliplatin and fluoropyrimidine combination chemotherapy for first-line treatment of advanced colorectal cancer: results of the randomised phase 3 MRC COIN trial. Lancet Oncol 12: $642-653$.

Dirican A, Kucukzeybek BB, Alacacioglu A, Kucukzeybek Y, Erten C, Varol U, Somali I, Demir L, Bayoglu IV, Yildiz Y, Akyol M, Koyuncu B, Coban E, Ulger E, Unay FC, Tarhan MO (2014) Do the derived neutrophil to lymphocyte ratio and the neutrophil to lymphocyte ratio predict prognosis in breast cancer? Int J Clin Oncol 20: 70-81.

Douillard J-Y, Oliner KS, Siena S, Tabernero J, Burkes R, Barugel M, Humblet Y, Bodoky G, Cunningham D, Jassem J, Rivera F, Kocákova I, Ruff P, Błasińska-Morawiec M, Šmakal M, Canon JL, Rother M, Williams R, Rong A, Wiezorek J, Sidhu R, Patterson SD (2013) Panitumumab-FOLFOX4 treatment and RAS mutations in colorectal cancer. N Engl J Med 369: 1023-1034.

Liu H, Liu G, Bao Q, Sun W, Bao H, Bi L, Wen W, Liu Y, Wang Z, Yin X, Bai Y, Hu X (2010) The baseline ratio of neutrophils to lymphocytes is associated with patient prognosis in rectal carcinoma. J Gastrointest Cancer 41: 116-120.

Proctor MJ, McMillan DC, Morrison DS, Fletcher CD, Horgan PG, Clarke SJ (2012) A derived neutrophil to lymphocyte ratio predicts survival in patients with cancer. Br J Cancer 107: 695-699.

Templeton AJ, McNamara MG, Šeruga B, Vera-Badillo FE, Aneja P, Ocaña A, Leibowitz-Amit R, Sonpavde G, Knox JJ, Tran B, Tannock IF, Amir E (2014) Prognostic role of neutrophil-to-lymphocyte ratio in solid tumors: a systematic review and meta-analysis. J Natl Cancer Inst; e-pub ahead of print 29 May 2014; doi:10.1093/jnci/dju124.

Walsh SR, Cook EJ, Goulder F, Justin TA, Keeling NJ (2005) Neutrophillymphocyte ratio as a prognostic factor in colorectal cancer. J Surg Oncol 91: $181-184$.

Walter SD (2002) Properties of the summary receiver operating characteristic (SROC) curve for diagnostic test data. Stat Med 21: 1237-1256.

This work is published under the standard license to publish agreement. After 12 months the work will become freely available and the license terms will switch to a Creative Commons AttributionNonCommercial-Share Alike 4.0 Unported License.

Supplementary Information accompanies this paper on British Journal of Cancer website (http://www.nature.com/bjc) 